\title{
BMJ Open Antiretroviral therapy for pregnant women living with HIV or hepatitis B: a systematic review and meta-analysis
}

\author{
Reed A Siemieniuk, ${ }^{1,2}$ Farid Foroutan, ${ }^{1}$ Reza Mirza, ${ }^{3}$ Jinell Mah Ming, ${ }^{4}$ \\ Paul E Alexander, ${ }^{1,5}$ Arnav Agarwal, ${ }^{6}$ Olufunmilayo Lesi, ${ }^{7}$ Arnaud Merglen, ${ }^{8}$ \\ Yaping Chang, ${ }^{1}$ Yuan Zhang, ${ }^{1}$ Hassan Mir, ${ }^{3}$ Elliot Hepworth, ${ }^{3}$ Yung Lee, ${ }^{9}$ \\ Dena Zeraatkar, ${ }^{1}$ Gordon H Guyatt ${ }^{1}$
}

To cite: Siemieniuk RA, Foroutan F, Mirza R, et al. Antiretroviral therapy for pregnant women living with HIV or hepatitis B: a systematic review and meta-analysis. BMJ Open 2017;7:e019022. doi:10.1136/ bmjopen-2017-019022

- Prepublication history and additional material for this paper are available online. To view please visit the journal (http:// dx.doi.org/10.1136/bmjopen2017-019022).

Received 7 August 2017 Accepted 17 August 2017

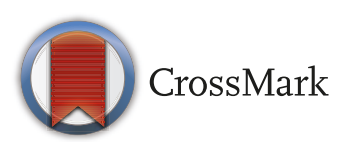

For numbered affiliations see end of article.

Correspondence to Professor Gordon H Guyatt; guyatt@mcmaster.ca

\section{ABSTRACT}

Objective To assess the impact of various antiretroviral/ antiviral regimens in pregnant women living with HIV or hepatitis $B$ virus (HBV).

Design We performed random effects meta-analysis for HIV-related outcomes and network meta-analysis for HBV outcomes, and used the Grading of Recommendations Assessment, Development and Evaluation (GRADE) framework to assess quality separately for each outcome. Data sources Embase and Medline to February 2017. Eligibility criteria For maternal outcomes, we considered randomised controlled trials (RCTs) comparing tenofovirbased regimens with those with alternative nucleoside/ nucleotide reverse transcriptase inhibitors (NRTIs). For child outcomes, we included RCTs and comparative observational studies of tenofovir-based regimens versus alternative NRTIs regimens or, for HBV, placebo.

Results Ten studies (seven RCTs) met the inclusion criteria for maternal and child outcomes, and an additional 33 studies (12 RCTs) met the inclusion criteria for HBVspecific outcomes. The most common comparison was tenofovir and emtricitabine versus zidovudine and lamivudine. There was no apparent difference between tenofovir-based regimens and alternatives in maternal outcomes, including serious laboratory adverse events (low certainty) and serious clinical adverse events (moderate certainty). There was no difference between NRTIs in vertical transmission of HIV: 1 more per 1000, 8 fewer to 10 more, low certainty; or vertical transmission of HBV: 7 fewer per 1000, 10 fewer to 38 more, moderate certainty. We found moderate certainty evidence that tenofovir/emtricitabine increases the risk of stillbirths and early neonatal mortality (51 more per 1000,11 more to 150 more) and the risk of early premature delivery at $<34$ weeks (42 more per 1000, 2 more to 127 more).

Conclusions Tenofovir/emtricitabine is likely to increase stillbirth/early neonatal death and early premature delivery compared with zidovudine/lamivudine, but certainty is low when they are not coprescribed with lopinavir/ritonavir. Other outcomes are likely similar between antiretrovirals. Trial registration number PROSPERO CRD42017054392

\section{BACKGROUND}

More than 17 million women are living with HIV, most of whom are of childbearing age. ${ }^{1}$
Strengths and limitations of this study

- We synthesise the best available evidence to inform choice of HIV and/or hepatitis B therapy for pregnant women.

- This review is linked to a BMJ Rapid Recommendations project. We conducted the review directed by a guideline panel that included patient representatives. This guideline panel provided detailed input with regard to the patients, interventions and outcomes, and the interpretation of the results from this review.

- We paid careful attention to what evidence could be appropriately pooled and which could not.

- The evidence for a likely increase of early premature delivery and neonatal mortality with tenofovir and emtricitabine comes mostly from a single study.

Every year, 1.4 million of these women experience pregnancies, which, without any intervention, carry a risk of vertical transmission to the infant of approximately $15 \%-45 \% .^{23}$ To reduce the risk of vertical transmission, approximately $80 \%$ of pregnant women living with HIV use antiretroviral therapy, primarily combination antiretroviral therapy (cART). ${ }^{4}$ The risk of vertical transmission is below $2 \%$ in high-income countries and below $5 \%$ in several low-income and middle-income countries when cART is universally available and routine HIV screening of pregnant mothers is provided.$^{5-7}$ Early initiation of cART may also reduce the risk of serious HIV-related events in all patients living with HIV ${ }^{89}$ which has resulted in the WHO recommending cART for all people living with HIV, including pregnant women. ${ }^{10}$

cART typically consists of two nucleoside/nucleotide reverse transcriptase inhibitors (NRTIs) - the 'backbone'-and a third antiretroviral agent. The most frequently used NRTI is tenofovir disoproxil 
fumarate (TDF), which is most often coformulated with another NRTI, emtricitabine (FTC) or lamivudine as a convenient once per day medication. Approximately $70 \%$ of persons taking cART use a tenofovir-based regimen, both in high-income and low-income and middle-income countries. ${ }^{11}$

In a November 2016 publication, the Promoting Maternal and Infant Survival Everywhere (PROMISE) study randomised pregnant women to either zidovudine (AZT) monotherapy or one of two cART arms with different NRTI backbones: tenofovir/FTC and AZT/ lamivudine, each combined with the protease inhibitor lopinavir, boosted with ritonavir (hereafter, $\mathrm{LPV} / \mathrm{r}$ ). ${ }^{12}$ The authors reported that both cART regimens reduced vertical transmission more effectively than AZT monotherapy. Notably, tenofovir/FTC, compared with AZT/ lamivudine, was associated with an increased risk of early premature labour, early neonatal death and a composite of severe adverse pregnancy outcomes. A subsequent systematic review concluded that tenofovir/FTC appears generally safe in pregnancy, but assumed equal credibility in randomised and observational studies by pooling evidence from all studies. ${ }^{13}$

NRTIs can also be used for indications other than HIV treatment. Tenofovir or lamivudine are often used in the third trimester to reduce the risk of vertical transmission of hepatitis B virus (HBV) ${ }^{14}$ HIV-negative women at risk for HIV, many of whom will become pregnant, may also use tenofovir/FTC for pre-exposure prophylaxis (PrEP) to reduce risk of HIV infection. ${ }^{15}$ The Joint United Nations Programme on HIV/AIDS (UNAIDS) has set a global target to increase uptake of PrEP to more than 3 million people by $2020 .{ }^{16}$

The WHO and the Centers for Disease Control and Prevention (CDC), despite being aware of the preliminary data from the PROMISE trial presented at a conference in $2015,{ }^{17}$ recommended tenofovir/FTC as first-line therapy for all pregnant women. ${ }^{10} 18$ We revisited this issue after publication of the full report ${ }^{12}$ that raised concerns about the safety of tenofovir/FTC in pregnancy. Our approach contrasts with a prior effort that pooled randomised controlled trials (RCTs) with far less trustworthy observational studies ${ }^{13}$ : our more standard approach deals with these two designs separately. Because of the high prevalence of hepatitis B and HIV coinfection and because the same medications are used for both conditions, we also include an evaluation of the impact of tenofovir versus alternative antivirals in pregnant women living with hepatitis B. This systematic review, along with a systematic review on patient values and preferences, ${ }^{19}$ informs a BMJ Rapid Recommendation ${ }^{11}$ (see box 1). The BMJ Rapid Recommendation initiative attempts to provide timely, unconflicted and trustworthy recommendations for clinical situations where new evidence might change practice. $^{20}$
Box 1 Linked articles in this BMJ Rapid

Recommendations cluster

Siemieniuk R, et al. Antiretroviral therapy in pregnant women living with HIV: a clinical practice guideline.

- BMJ Rapid Recommendation

Lytvyn L, et al. Values and preferences of women living with HIV who are pregnant or considering pregnancy on choice of antiretroviral therapy during pregnancy (cosubmitted).

- A systematic review of values and preferences

MAGICapp

- Expanded version of the evidence with multilayered recommendations, evidence summaries and decision aids for use on all devices

https://www.magicapp.org/goto/guideline/VLpr5E

\section{METHODS}

\section{Protocol}

We conducted this systematic review based on a registered protocol (PROSPERO CRD42017054392).

\section{Patient involvement}

As with all $B M J$ Rapid Recommendations, patients were included in all stages of the research production (see box 2).

\section{Information sources}

Our review used three separate search strategies. First, we searched Medline and Embase from 1 January 1996 to 13 January 2017 for observational studies and RCTs that compared a tenofovir-based cART regimen with another regimen with the same non-NRTI antiretroviral in pregnant women, using a mix of keywords and medical subject headings (MeSH) terms for HIV and pregnancy and NRTIs (online supplementary appendix 1a). Second, anticipating that for many maternal outcomes there would be only low-quality or very low-quality evidence if we included only direct evidence from pregnant women, we searched for RCTs of non-pregnant adults living with HIV initiating cART with a tenofovir-based regimen or an alternative NRTI-regimen that included the same non-NRTI antiretroviral(s). We updated a comprehensive search conducted on 7 July $2015 .{ }^{21}$ We searched from 7 July 2015 to 17 February 2017 and used a mix of MeSH and keywords for HIV and antiretrovirals and RCTs (online supplementary appendix $1 \mathrm{~b}$ ). We also searched the abstracts of recent major conferences, including the

\section{Box 2 Patient involvement}

Three women living with HIV, two of whom had children after being diagnosed with HIV and another who is considering having children in the future, participated in the panel. The community representatives received personalised training and support to optimise contributions throughout the guideline development process. These women helped choose the outcomes that were most important to them, all of which were included in our review. The patient panellists approved the review protocol and helped guide interpretation of the results. 
Conference of Retroviruses and Opportunistic Infections, the International AIDS Society Conference and the International AIDS Conference on 17 February 2017.

To inform outcomes specific to pregnant women living with chronic HBV infection, we searched for comparative observational studies and RCTs of tenofovir, lamivudine or FTC in pregnant women living with HBV. We built on a systematic search conducted on 11 September 2014. ${ }^{22}$ We searched Medline and Embase from 1 January 2014 to 14 January 2017. We used a combination of keywords and MeSH terms for pregnancy and HBV and antivirals (online supplementary appendix 1c).

We also searched reference lists of all included studies, systematic reviews and relevant guidelines. We searched ClinicalTrials.gov for additional studies on 17 February 2017 , including unpublished studies. We did not have any language restrictions and had two reviewers fluent in the language of publication assess for inclusion and abstract data if deemed eligible.

\section{Study selection}

For child outcomes, we included observational studies and RCTs that compared tenofovir with alternative NRTI regimens in pregnant women. We included studies on women taking NRTIs for PrEP, for treatment of hepatitis B or for HIV infection, in combination with other antiretrovirals as long as the non-NRTI antiretrovirals were the same in both arms. Because for several critical outcomes specific to the mother we anticipated finding no direct evidence or the evidence would be of very low certainty, we also included RCTs that compared tenofovir-based regimens with alternative NRTIs in non-pregnant adults living with HIV. We considered evidence from pregnant women alone before including evidence from non-pregnant adults. For child outcomes, we included studies of PrEP (tenofovir/FTC vs placebo). For outcomes specific to women also living with HBV, we included observational studies and RCTs that compared tenofovir, FTC or lamivudine against each other or, because we anticipated that there would be few head-to-head studies, a control (no antiviral treatment). We excluded studies of NRTIs that are not in widespread use or are not used for HIV infection, including stavudine, didanosine, zalcitabine, adefovir and entecavir. Observational studies included cohort, case-control and any other observational study type that attempted a direct and coincident comparison between any two of the eligible interventions.

Reviewers screened all titles and abstracts independently and in duplicate. If either reviewer felt that a study might meet inclusion criteria, two reviewers independently assessed the full text. Reviewers resolved disagreements through discussion.

\section{Data collection}

Two reviewers independently abstracted data and resolved conflicts by discussion. When data were only available in a figure, we digitised the figure.

\section{BMJ Rapid Recommendation process}

The semi-independent Rapid Recommendation panel chose outcomes they felt were most likely to influence patient decisions between NRTI regimens; they also identified subgroups in whom effects might differ. As with all BMJ Rapid Recommendations, ${ }^{23-25}$ the panel was free from financial conflicts, and intellectual and professional conflicts were minimised. ${ }^{20}$ The panel included three women living with HIV, clinical experts (two obstetricians, four paediatricians, three infectious diseases specialists, a pharmacist, a hepatologist and a primary care physician with substantial experience treating HIV) and five Grading of Recommendations Assessment, Development and Evaluation (GRADE) methodologists. ${ }^{25}$ Panellists resided in Africa, Australasia, Europe, North America and South America. The guideline panel provided critical oversight to the review and identified populations, subgroups and outcomes of interest. Panel members provided input at all stages of the systematic review. The patient panel members led the interpretation of the results based on what they expected the typical patient values and preferences to be, as well as the variation between patients. A parallel systematic review of patient values and preferences was also conducted to help with interpretation. ${ }^{19}$

\section{Summary measures}

Maternal outcomes included mortality, acceptability (we used drug discontinuation rates as a surrogate), clinical adverse events (grade 2 or higher), ${ }^{26}$ laboratory adverse events (grade 2 or higher), detectable viral load 6 months after starting cART as a proxy for viral load at delivery, AIDS-defining illnesses, hepatitis B flares and development of HBV resistance to one or more antivirals. When we included data from RCTs in non-pregnant adults, we used the endpoint closest to 26 weeks after enrolment to approximate the timeline of a woman-starting cART at the beginning of the second trimester. Fetal outcomes included a composite of stillbirth after 20 weeks' gestational age (GA) and early neonatal mortality within the first week, spontaneous abortion, HIV transmission, prematurity $<37$ weeks, early prematurity $<34$ weeks, serious birth defects, low birth weight $<2500 \mathrm{~g}$, very low birth weight $<1500 \mathrm{~g}$, neonatal adverse laboratory event (grade 2 or higher), long-term child growth/development and HBV transmission. We combined stillbirths with early neonatal mortality because of a similar pathophysiology (most early neonatal deaths are caused by pregnancy-related factors) and because we believe that most women would place a similar value on the two events.

\section{Risk of bias and quality of evidence}

We used a modified Cochrane Collaboration tool to assess risk of bias for RCTs, ${ }^{27}$ which substitutes response options of 'probably low risk' or 'probably high risk' for unclear; empirical evaluation has shown that reviewers can make these judgements accurately. ${ }^{28}$ Ultimately, we collapsed the low and probably low, and high and probably high 
risk, for presentation. Two reviewers assessed risk of bias independently and resolved disagreements through discussion. We used a modified Ottawa-Newcastle instrument for assessing risk of bias for observational studies. ${ }^{29}$

The GRADE approach provided the framework for rating the certainty of evidence for each outcome. ${ }^{30}$ Evidence from RCTs started at high certainty, whereas evidence from observational studies started at low certainty. Concerns with risk of bias, inconsistency, indirectness, imprecision and publication bias lowered certainty. We considered the bodies of evidence from RCTs and observational studies separately.

For the outcomes specific to HBV, we used the GRADE approach for rating certainty of network effect estimates obtained from a network meta-analysis. ${ }^{31}$ In brief, we rated the certainty of evidence for direct comparisons using the standard GRADE approach. For indirect comparisons, we rated evidence from the dominant first-order loop by first taking the lowest certainty of the direct comparisons. We then considered further rating down if there were concerns with intransitivity. ${ }^{31}$ For mixed estimates (those that included both indirect and direct evidence), we started with the higher of the two certainty ratings and rated down certainty for imprecision or incoherence between the indirect and direct effect estimates.

\section{Subgroups and sensitivity analyses}

We planned subgroup analyses if there were at least two studies per group (online supplementary appendix 2). We performed a post hoc sensitivity analysis for pregnancy loss and early infant death as well as premature labour <34 weeks, including PROMISE participants randomised to AZT/lamivudine prior to the introduction of the tenofovir/FTC arm because of concerns that there were fewer events than expected in the AZT/lamivudine arm in the latter part of the study.

\section{Synthesis of results}

We used random effects meta-analysis of risk ratios (RRs) and calculated 95\% CIs with the DerSimonian and Laird approach. When events were rare across all studies $(<2 \%)$, we performed meta-analysis directly with the Peto method unless one or more studies had zero events in both arms, in which case we used risk differences (RD) directly. We planned assessment of publication bias with visual inspection of funnel plots for outcomes with 10 or more studies. We present evidence that led to the highest quality using the GRADE framework-for all outcomes, looking first for evidence from RCTs of pregnant women, but if that evidence was either not available or proved of low or very low certainty, then also considering evidence from RCTs of non-pregnant adults and observational studies of pregnant women living with HIV.

For the comparisons of antivirals for HBV infection, we anticipated that there would be few if any direct comparisons between antivirals and therefore performed a network meta-analysis within a frequentist framework using RRs. We added 0.5 events to both arms if one arm had zero events and excluded trials with zero events in both arms because CIs could not be calculated. Direct comparisons were also analysed with standard pairwise DerSimonian and Laird meta-analysis. We used the back-calculation and node splitting methods to estimate the RR and CIs from indirect and direct evidence and to assess for incoherence. Inconsistency was assessed for each pairwise comparison by visual inspection of forest plots and the $\mathrm{I}^{2}$ statistic for heterogeneity. We also considered the global $\mathrm{I}^{2}$ for network meta-analyses. ${ }^{32}$ We used RevMan V.5.3 for meta-analyses of direct comparisons and Stata V.13 and the netmeta package in $\mathrm{R}$ ( $\mathrm{R}$ project) for network meta-analyses.

We present all outcomes as absolute effects, either calculated directly or by multiplying the RR by the baseline risk. Where possible, we apply the relative risk calculated from RCTs to a baseline risk from observational studies. ${ }^{33}$ For outcomes in which trustworthy observational data were not identified, we used the pooled baseline risk from the control group. The Rapid Recommendation panel suggested outcomes in which they expected baseline risk to differ between settings (eg, the panel believed vertical transmission of HBV would be lower in high-resourced health systems than lower resource settings).

\section{RESULTS}

We screened 2750 studies in the primary search for comparative studies in pregnant women and included 10 studies (online supplementary appendix 3a). All studies compared a tenofovir-based regimen with placebo or alternative NRTI-based regimens in pregnant women: seven were RCTs (three included women living with HIV, ${ }^{12} 3435$ three evaluated tenofovir/FTC for PrEP in HIV-negative women, ${ }^{15} 3637$ and one evaluated tenofovir alone in pregnant women with $\mathrm{HBV}$ infection ${ }^{38}$ ) and three were observational cohort studies of HIV-positive women $^{39-41}$ (table 1). Two of the potentially eligible PrEP RCTs had very low compliance (less than $33 \%$ ), and we therefore excluded them from further consideration. ${ }^{36} 37$ The PrEP RCT that we included had greater than $60 \%$ compliance, discouraged pregnancy, tested for pregnancy monthly and discontinued the study medications when pregnancy was detected (at an average of 35 days' GA). ${ }^{15}$ Given the very early and limited exposure to antiretroviral medication, we included this study only for the outcome of stillbirth. The RCT of tenofovir versus placebo in pregnant women with HBV infection initiated therapy at 32 weeks' gestation. ${ }^{38}$ Given the limited late exposure to the drugs, we included these results only for the outcome of stillbirths, early neonatal deaths and low birth weight. At the request of the $B M J$ Rapid Recommendation panel, ${ }^{11}$ we also included evidence from the Antiretroviral Pregnancy Registry for the outcome of birth defects. ${ }^{42}$ The registry is a frequently updated non-comparative database that tracks the incidence of birth defects in mothers who have taken antiretrovirals. 


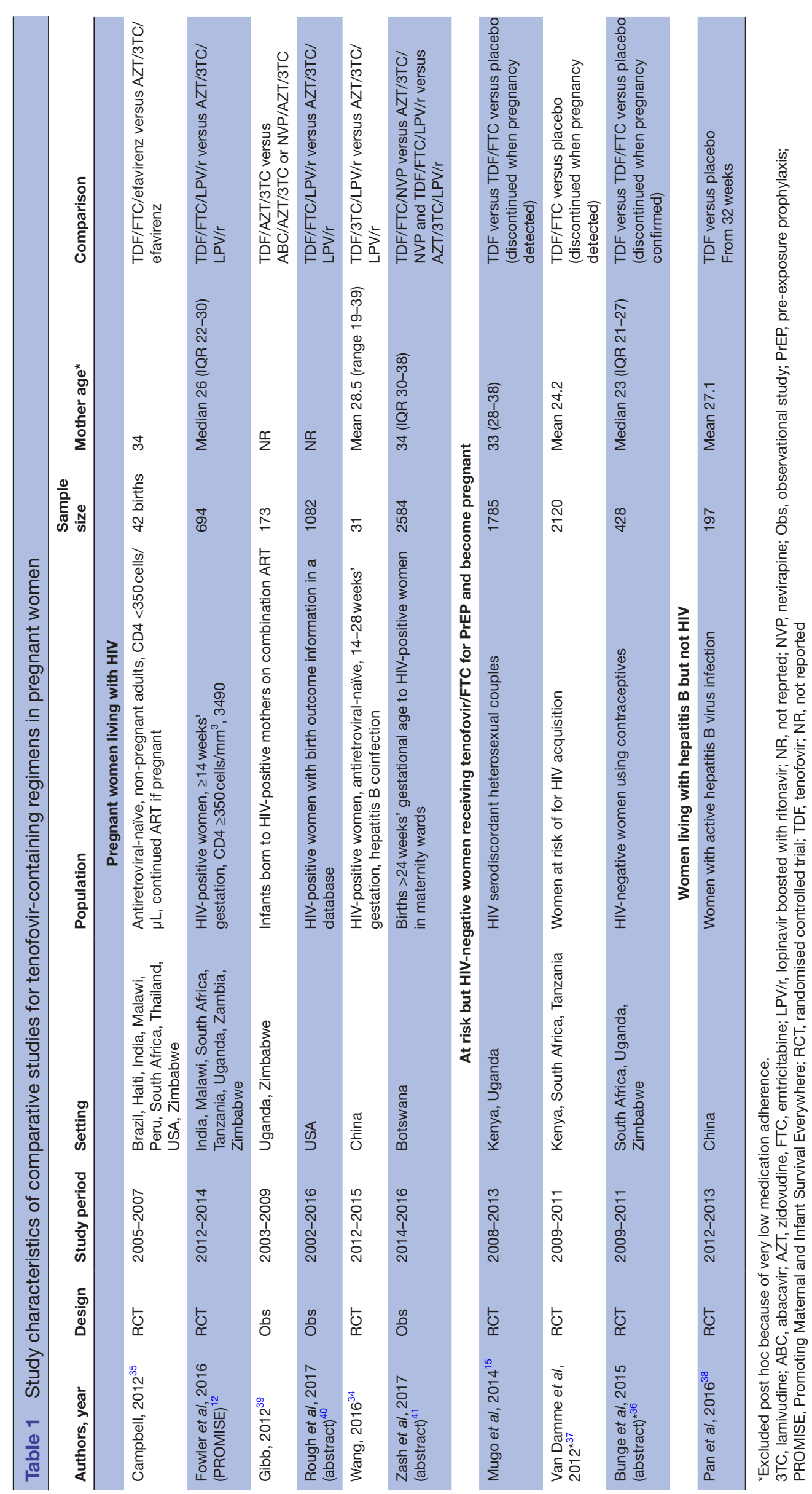

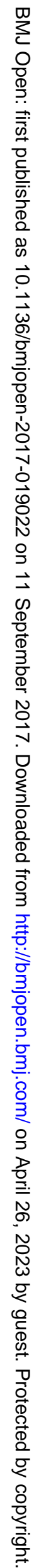


RCTs that enrolled pregnant women provided evidence at low risk of bias. The main limitation of the RCTs was the lack of blinding in the PROMISE trial ${ }^{12}$ and two smaller RCTs. ${ }^{34} 38$ The PROMISE trial was also stopped early, but that decision was based on the reduction in vertical transmission of cART compared with AZT monotherapy and therefore should not bias comparison of tenofovir-based cART versus alternative NRTI-based cART, the focus of this review. ${ }^{12}$ The PROMISE trial randomised 823 women, most in Africa, to the comparison of interest. All the observational studies were at high risk of bias because the analyses did not control for most expected key confounders (eg, socioeconomic status and year of cART initiation).

To inform outcomes specific to the mothers in which direct evidence from pregnant women provided only low-quality or very low-quality evidence, we considered indirect evidence from RCTs of tenofovir-based regimens versus alternative NRTI-based regimens in non-pregnant HIV-positive adults (table 2). We screened 297 studies and ultimately included eight RCTs from nine publications with 5353 participants (online supplementary appendix 3b). ${ }^{12} 3543-49$ Four RCTs with 2316 participants compared tenofovir/FTC with AZT/lamivudine, ${ }^{12} 354346$ and four with 3037 participants with abacavir/lamivudine. These RCTs were limited primarily by lack of allocation concealment ( 4 of 8 ) and lack of blinding ( 5 of 8 ) (online supplementary appendix 4a).

\section{Maternal outcomes}

Acceptability

Pooled evidence for discontinuation rates from seven RCTs $(n=4198)$ including non-pregnant adults proved of very low certainty due to inconsistency, indirectness because evidence is from non-pregnant adults, and imprecision (online supplementary appendix 5a). Higher certainty evidence addressing acceptability came from medication discontinuation rates in the PROMISE trial, ${ }^{12}$ in which there was no important difference between groups: 15 $(4.2 \%, \mathrm{n}=356)$ discontinued in the tenofovir/FTC group and $10(2.8 \%, \mathrm{n}=360)$ discontinued in the AZT/lamivudine group (RD 15 more per 1000 discontinued with tenofovir/FTC, CI 9 fewer to 65 more; table 3).

\section{Mortality}

For mortality, the PROMISE trial ${ }^{12}$ and pooled estimates from RCTs of pregnant women provided moderate certainty evidence of no important difference between alternative cART regimens. No women in the PROMISE trial died ${ }^{12}$ : RD 0 per 1000 , CI 11 fewer to 11 more. There was no apparent difference between tenofovir/FTC $(1.4 \%, \mathrm{n}=2337)$ and alternative NRTIs $(1.6 \%, \mathrm{n}=2313)$ in mortality in seven RCTs $(n=4650)$ that included non-pregnant adults (RD 2 fewer per 1000 with tenofovir, CI 6 fewer to 2 more; online supplementary appendix 5b).

\section{Clinical maternal adverse events}

Although low certainty evidence (very serious imprecision) from the PROMISE trial suggested no difference between groups ${ }^{12}$ higher certainty evidence for clinical maternal adverse events comes from pooled estimates from RCTs of non-pregnant adults. Six RCTs reported adverse effects; three compared AZT/lamivudine versus tenofovir/FTC $(\mathrm{n}=2139)$, and three abacavir/lamivudine versus tenofovir/FTC $(\mathrm{n}=2343)$. Results suggested a subgroup difference between AZT/lamivudine and abacavir/lamivudine, with relatively more adverse events in the abacavir/lamivudine group than in the AZT/ lamivudine group ( $\mathrm{p}$ for interaction=0.009) (figure 1). Clinical adverse effects were similar for tenofovir/FTC $(26.8 \%, \mathrm{n}=1061)$ and AZT/lamivudine $(26.3 \%, \mathrm{n}=1078)$ : RR 1.00 , CI 0.90 to $1.12, \mathrm{I}^{2}=0 \%$; RD 0 per 1000 (table 3 ). There were fewer clinical adverse events in the tenofovir/ FTC group $(14.1 \%, \mathrm{n}=1173)$ than the abacavir/lamivudine group (19.6\%, $\mathrm{n}=1170$ ): RR 0.72 , CI 0.60 to 0.86 , $\mathrm{I}^{2}=0 \%$; RD 8 fewer per 1000; moderate certainty due to indirectness. Pain or discomfort $(6.0 \%)$ and pruritus $(2.3 \%)$ accounted for most of the difference in one study that combined each with atazanavir/ritonavir. ${ }^{48}$

\section{Maternal laboratory adverse events}

Four RCTs $(n=2217)$, three of which were in non-pregnant adults, reported fewer grade 2 or higher laboratory adverse events with tenofovir/FTC than alternatives, but the evidence proved lower certainty evidence than the PROMISE $^{12}$ trial alone (inconsistency and indirectness) (figure 2). In the PROMISE trial ${ }^{12}$ there was no apparent difference in laboratory adverse events between tenofovir/FTC $(10.9 \%, \mathrm{n}=329)$ and AZT/lamivudine $(12.9 \%$, $\mathrm{n}=333$ ): RR 0.85 , CI 0.56 to 1.28 ; RD 19 fewer per 1000 (table 3).

\section{Undetectable viral load 6 months after starting cART}

The PROMISE study did not provide data informing viral load outcomes at birth. ${ }^{12}$ We therefore examined indirect evidence in non-pregnant adults living with HIV initiating cART: failure to suppress HIV viral load at 6 months after starting therapy to approximate viral load suppression at delivery for a pregnant woman initiating cART at the start of the second trimester. The pooled results from six RCTs ( $n=4220$ ) suggested no difference between tenofovir-based cART $(19.5 \%, \mathrm{n}=2126)$ and alternative NRTIs (22.2\%, n=2094): RR 0.93, CI 0.71 to $1.23 ; \mathrm{I}^{2}=77 \%$; RD 16 fewer per 1000 (figure 3, table 3).

\section{Child outcomes}

\section{Stillbirth and early neonatal mortality}

The evidence from the PROMISE trial ${ }^{12}$ and two smaller RCTs reported $21(6.3 \%, \mathrm{n}=334)$ stillbirths and early infant deaths in the tenofovir/FTC arm and 5 $(1.4 \%, \mathrm{n}=348)$ in the AZT/lamivudine arm (pooled RR 4.40 , CI 1.75 to $11.01 ; \mathrm{I}^{2}=0 \%$; figure 4 ). Observational evidence suggests that the baseline risk of stillbirth and early neonatal mortality is approximately 15 per 1000 in high-income countries ${ }^{50}$ and approximately 69 per 1000 in low-income countries. ${ }^{51}$ The best estimate of the increase in stillbirths and neonatal mortality is therefore 


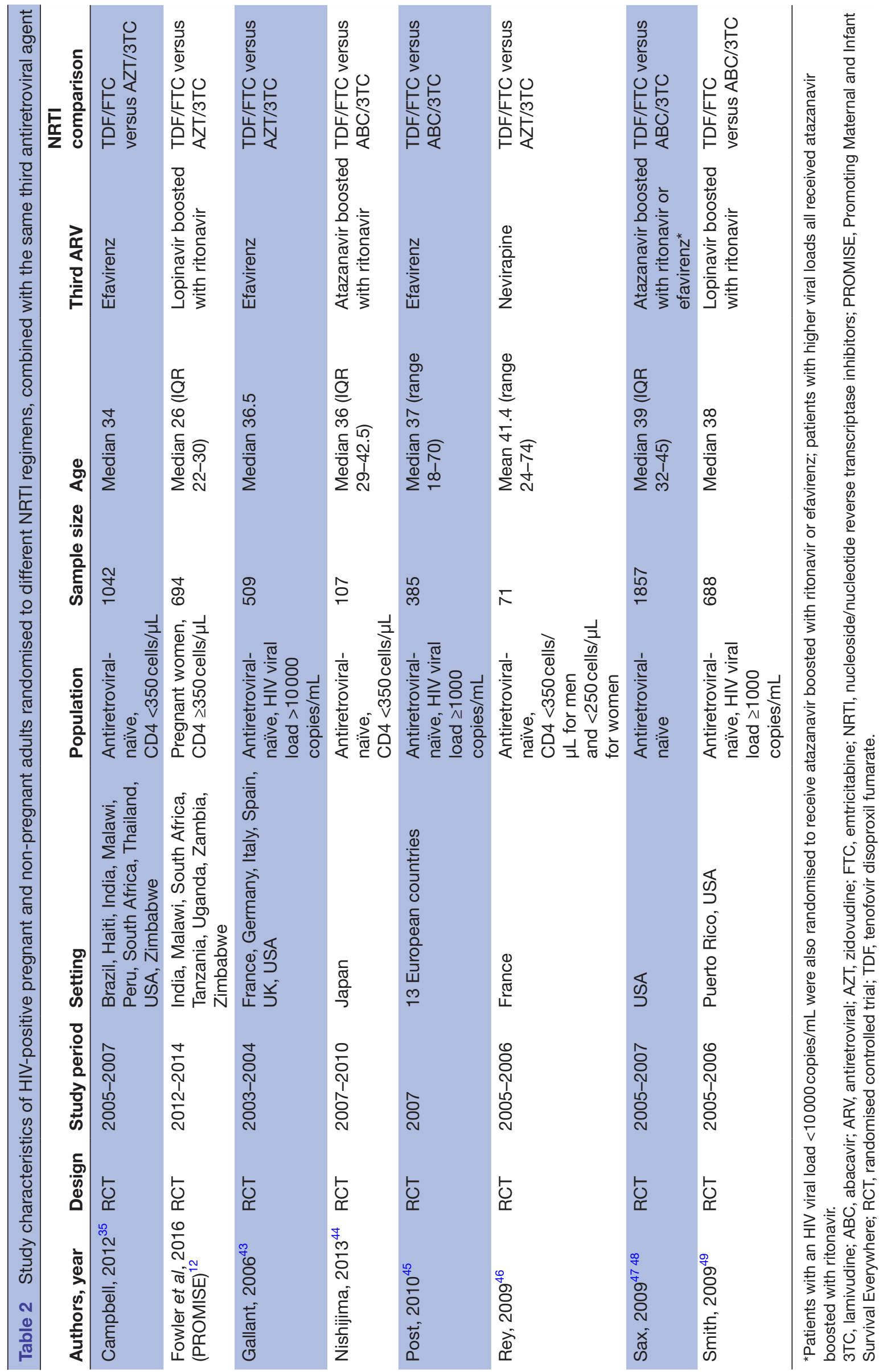




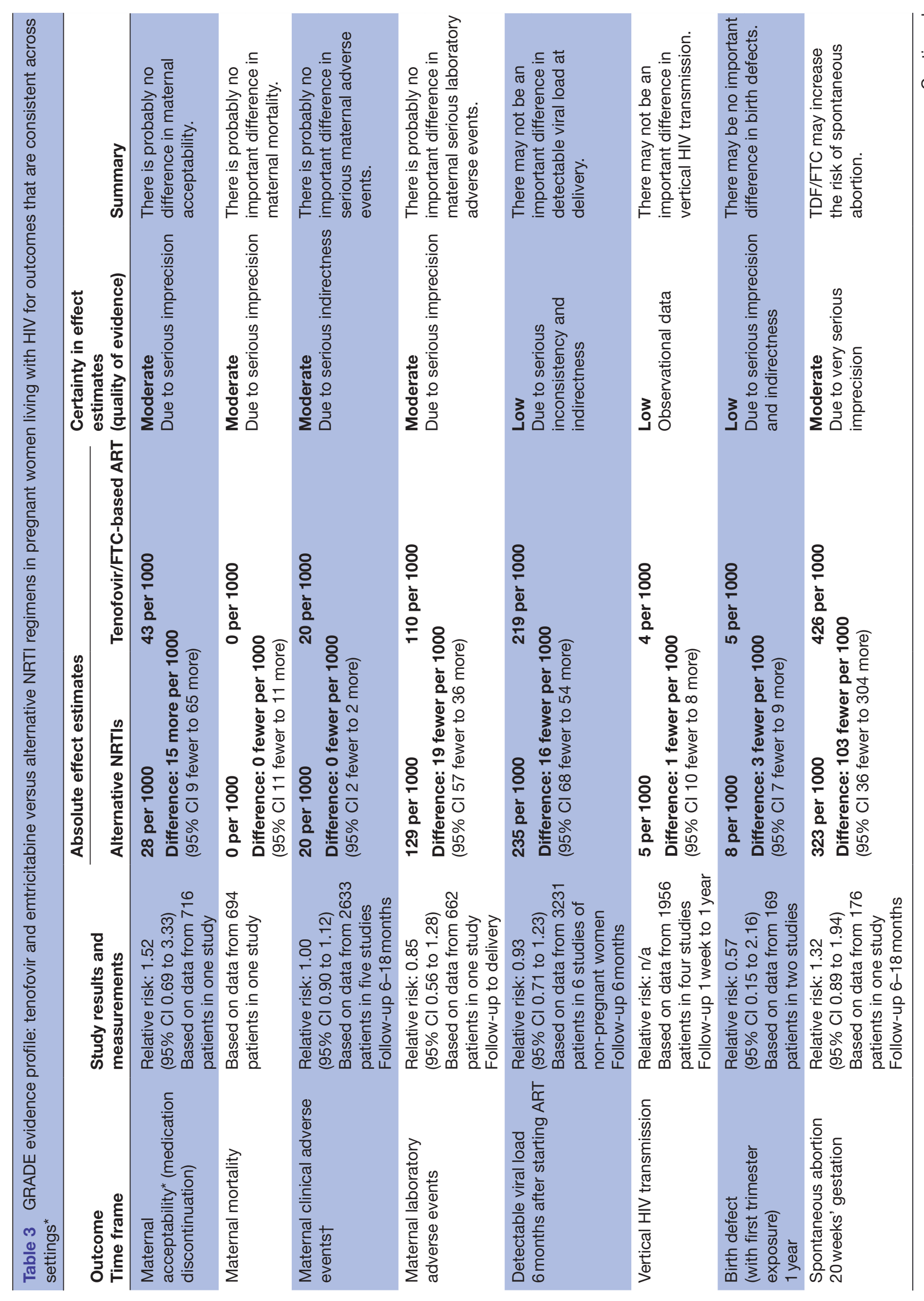

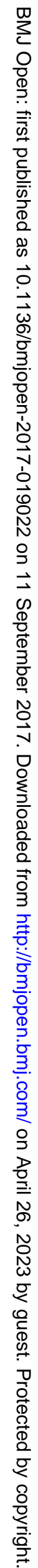




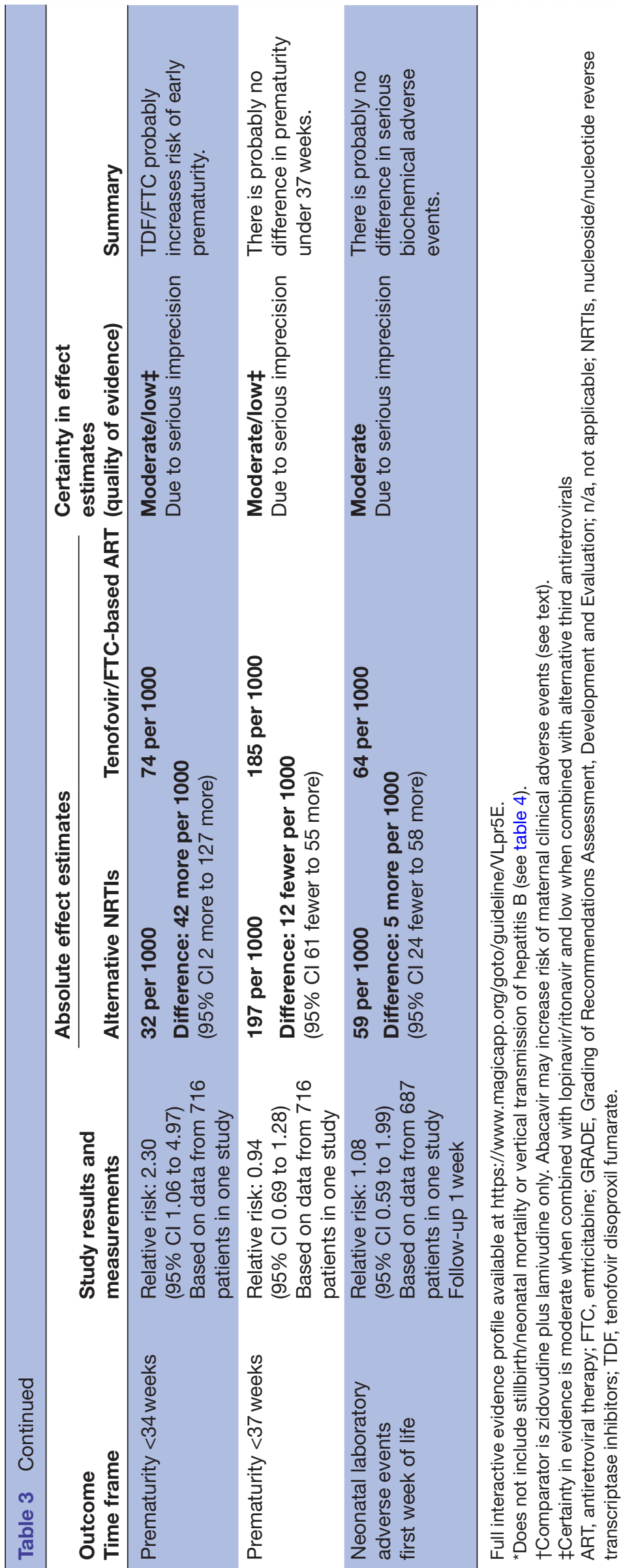




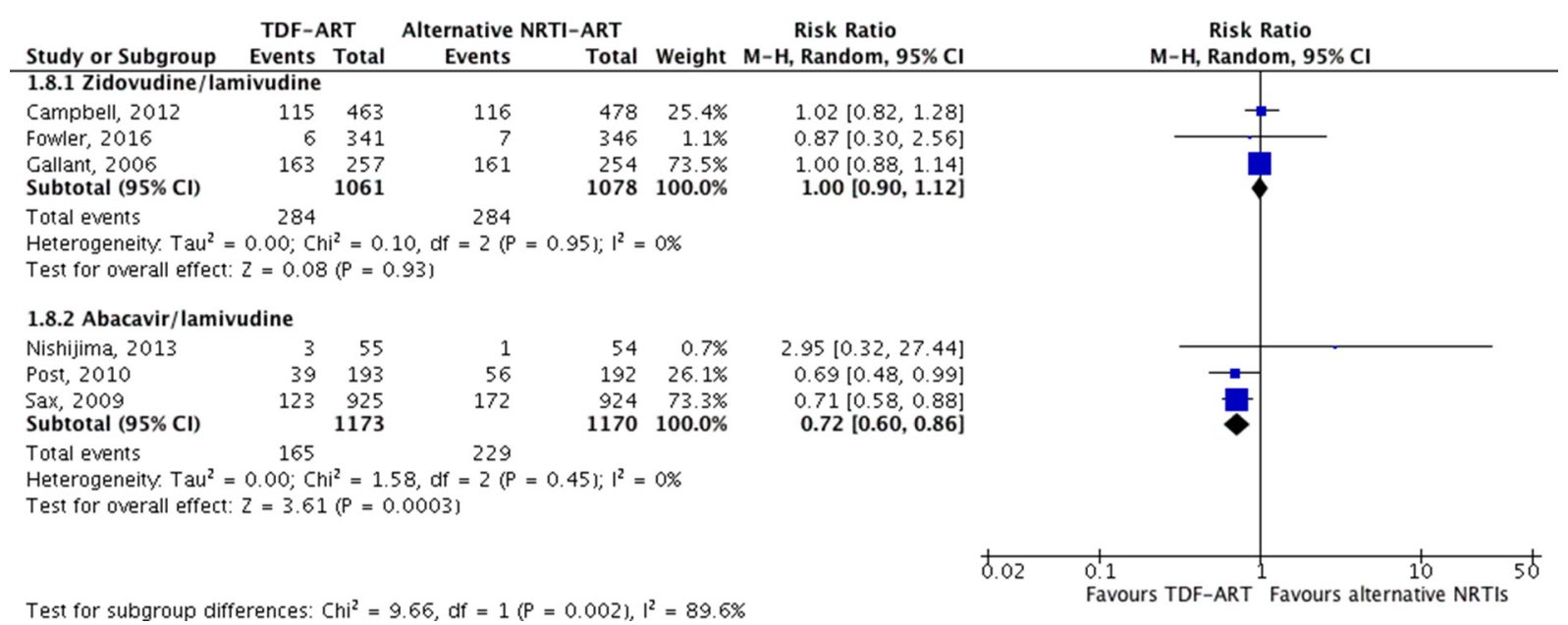

Figure 1 Forest plot of the risk ratio for clinical adverse events (data from randomised trials in non-pregnant adults except Fowler et $\mathrm{al}^{12}$ ). ART, antiretroviral therapy; M-H, Mantel-Haenszel; NRTI, nucleoside/nucleotide reverse transcriptase inhibitor; TDF, tenofovir disoproxil fumarate.

51 more per 1000 in low-income settings and 235 more per 1000 in high-income settings (table 4 ). In a post hoc sensitivity analysis that included PROMISE participants ${ }^{12}$ randomised to AZT/lamivudine prior to the introduction of the tenofovir/FTC arm, the results remained statistically significant.

Observational studies reported conflicting results (online supplementary appendix 5c). One suggested a higher rate of stillbirth and early neonatal mortality in tenofovir-based regimens than in alternative regimens (combined in a triple NRTI regimen with AZT/lamivudine $)^{39}$; two others found similar results in tenofovir-based and alternative NRTI regimens (combined with either $\mathrm{LPV} / \mathrm{r}$ or nevirapine $)^{40}{ }^{41}$; and one reported a lower rate of stillbirths and early neonatal mortality (combined with nevirapine).$^{41}$ No study controlled for most of the critical confounders such as socioeconomic status, immune/ disease status and cointerventions. Pooled results from these four observational studies suggested no difference between tenofovir-based and alternative regimens, but with a wide CI: RR 0.92, CI 0.52 to $1.64 ; \mathrm{I}^{2}=68 \%$ (online supplementary appendix 5c). Thus, the evidence from observational studies is of very low certainty due to the observational design, imprecision, inconsistency and risk of bias.

\section{Vertical transmission of HIV}

Two observational studies including 1850 patients $^{12} 39$ and two small RCTs with 75 patients $^{34}{ }^{35}$ reported vertical transmission of HIV. The PROMISE trial did not report vertical transmission in the groups as randomised, and therefore we considered it an observational study for this outcome $^{12}$; there were no other transmission events in any of the other studies. There were two $(0.4 \%, \mathrm{n}=472)$ transmission events in the TDF/FTC-based cART group and seven $(0.5 \%, \mathrm{n}=1484)$ in the alternative NRTI groups: RD 1 fewer per 1000, CI 10 fewer to 8 more; low certainty due to observational design (online supplementary appendix $5 d)$.

\section{Birth defects}

The PROMISE trial and a study of PrEP did not detect any difference in birth defects ${ }^{12}{ }^{15}$ : RR 1.05 , CI 0.68 to 1.62 , RD 0 per 1000 , CI 3 fewer to 5 more, moderate certainty because of imprecision. However, women in the PROMISE trial were enrolled at a median of 26 weeks' gestation (IQR 21-31) ${ }^{12}$; thus, the evidence has little or no bearing on exposure in the first trimester. Evidence from two small RCTs with ART exposure in the first trimester did not find any apparent difference in birth defects between tenofovir and alternatives ${ }^{1535}$ : RR 0.57 , CI 0.15 to

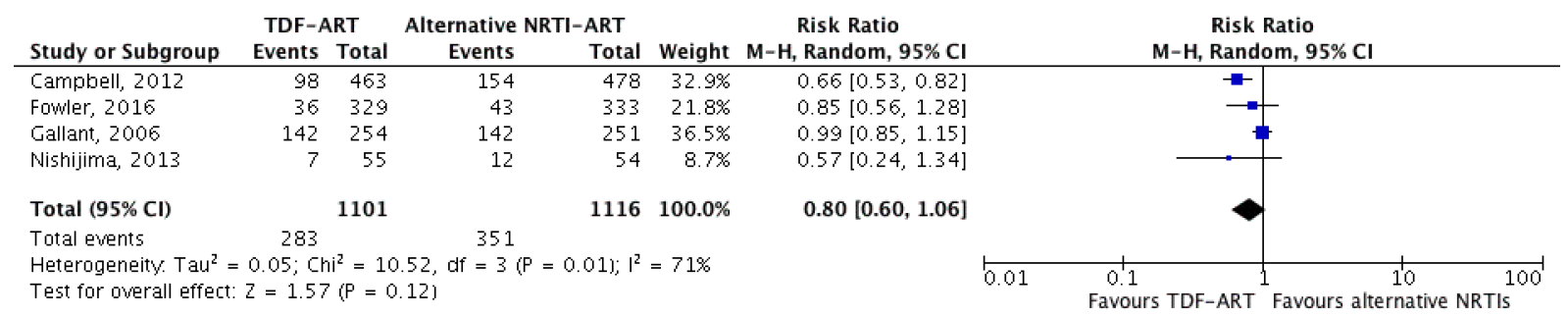

Figure 2 Forest plot of the risk ratio for laboratory adverse events (data from randomised trials in non-pregnant adults except Fowler et $\mathrm{al}^{12}$ ). ART, antiretroviral therapy; M-H, Mantel-Haenszel; NRTI, nucleoside/nucleotide reverse transcriptase inhibitor; TDF, tenofovir disoproxil fumarate. 


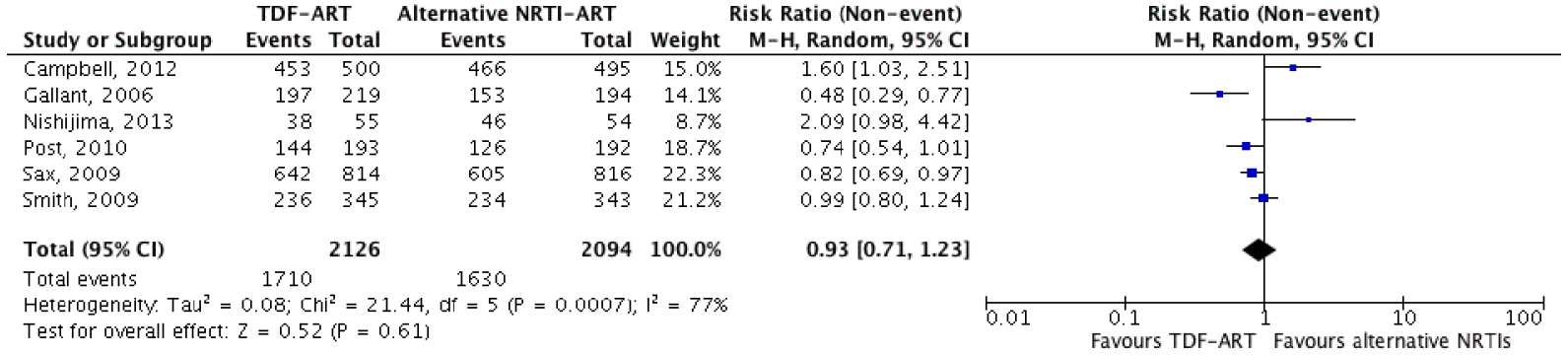

Figure 3 Forest plot of risk ratio for detectable serum viral load 26 weeks after antiretroviral initiation as a proxy for viral load at time of delivery (data from randomised trials of non-pregnant adults). ART, antiretroviral therapy; M-H, Mantel-Haenszel; NRTI, nucleoside/nucleotide reverse transcriptase inhibitor; TDF, tenofovir disoproxil fumarate.

2.16; RD 3 fewer per 1000 with TDF-ART, CI 7 fewer to 9 more (table 3). Two observational studies suggested that the overall birth defect rate might be lower with tenofovir-based ART than with alternative NRTIs - a result that was driven by the Antiretroviral Pregnancy Registry, ${ }^{42}$ that relies on voluntary reporting and dates back to 1989: RD 9 fewer per 1000, CI 16 fewer to 2 fewer; very low certainty due to the observational design, imprecision, and risk of bias (online supplementary appendix 5e).

\section{Spontaneous abortion}

The PROMISE trial did not report any spontaneous abortions, but did not enrol participants prior to 14 weeks' gestation and more than $75 \%$ of women were enrolled after 20 weeks' gestation. ${ }^{12}$ Evidence from an RCT of HIV PrEP suggested that tenofovir combined with FTC may increase the risk of pregnancy loss, $91 \%$ of which were spontaneous abortion: $42.5 \%$ (34 in 80 pregnancies) with tenofovir/FTC vs $32.3 \%$ (31 in 96 pregnancies) with placebo; RD: 103 more per 1000, CI 36 fewer to 304 more (table 3). ${ }^{15}$ Evidence from one observational study was consistent but did not increase certainty (online supplementary appendix 5f). ${ }^{39}$

\section{Prematurity at $<34$ and $<37$ weeks' gestation}

The PROMISE trial alone provided the highest quality evidence for prematurity. ${ }^{12}$ There was an increase in early prematurity <34 weeks' gestation with tenofovir/FTC (6.0\%, $\mathrm{n}=335)$ compared with AZT/lamivudine $(2.6 \%$, $\mathrm{n}=346)$ : RR 2.30, CI 1.06 to 4.97; RD 42 more per 1000 (table 3). All 35 infants were born after 34 weeks' gestation in one other RCT. ${ }^{34}$ The results were similar in a sensitivity analysis that included PROMISE ${ }^{12}$ participants randomised to AZT/lamivudine prior to the introduction of the tenofovir/FTC arm. There was no apparent difference in prematurity at $<37$ weeks' gestation between tenofovir/FTC $(18.5 \%, \mathrm{n}=335)$ and AZT/lamivudine $(19.7 \%$, $\mathrm{n}=346)$ : RR 0.94, CI 0.69 to 1.28 ; RD 12 fewer per 1000 (table 3$)$.

Three observational studies that included four comparisons $(n=3878)$ suggested that tenofovir-based cART may be associated with reduced risk of premature delivery $<37$ weeks, but certainty in evidence is very low because of the observational study design and in addition risk of bias from failure to control for key confounders (online supplementary appendix $5 \mathrm{~g}) .{ }^{39-41}$ Similarly, there was only very low-quality evidence from the same observational studies addressing very early or early premature delivery (online supplementary appendix 5h).

\section{Low and very low birth weight}

The PROMISE trial alone provides the highest quality evidence for low and very low birth weight. ${ }^{12}$ There was no apparent increase in low birth weight $<2500 \mathrm{~g}$ with tenofovir/FTC $(16.9 \%, \mathrm{n}=301)$ and AZT/lamivudine (20.4\%, n=319): RR 0.83, CI 0.60 to 1.16 ; RD 35 fewer per 1000; moderate certainty because of imprecision. There were more neonates born weighing $<1500 \mathrm{~g}$ with tenofovir/FTC $(2.1 \%, \mathrm{n}=335)$ than with AZT/lamivudine $(0.6 \%, \mathrm{n}=346)$ : RR 3.61, CI 0.76 to 17.28 ; RD 16 more per 1000; moderate certainty due to imprecision. One additional observational study did not improve the certainty in either outcome (online supplementary appendix $5 \mathrm{i}$ and $\mathrm{j}$ ).

\section{Neonatal laboratory adverse events}

The best evidence is provided by the PROMISE trial alone. ${ }^{12}$ There was no difference in grade 3 or higher

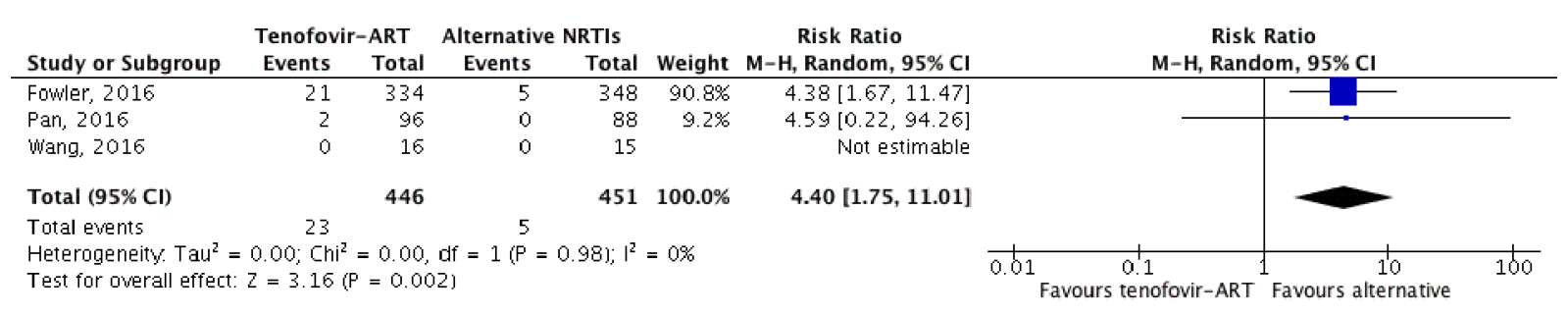

Figure 4 Forest plot of risk ratio for stillbirth and early neonatal mortality from randomised controlled trials. ART, antiretroviral therapy; M-H, Mantel-Haenszel; NRTI, nucleoside/nucleotide reverse transcriptase inhibitor. 


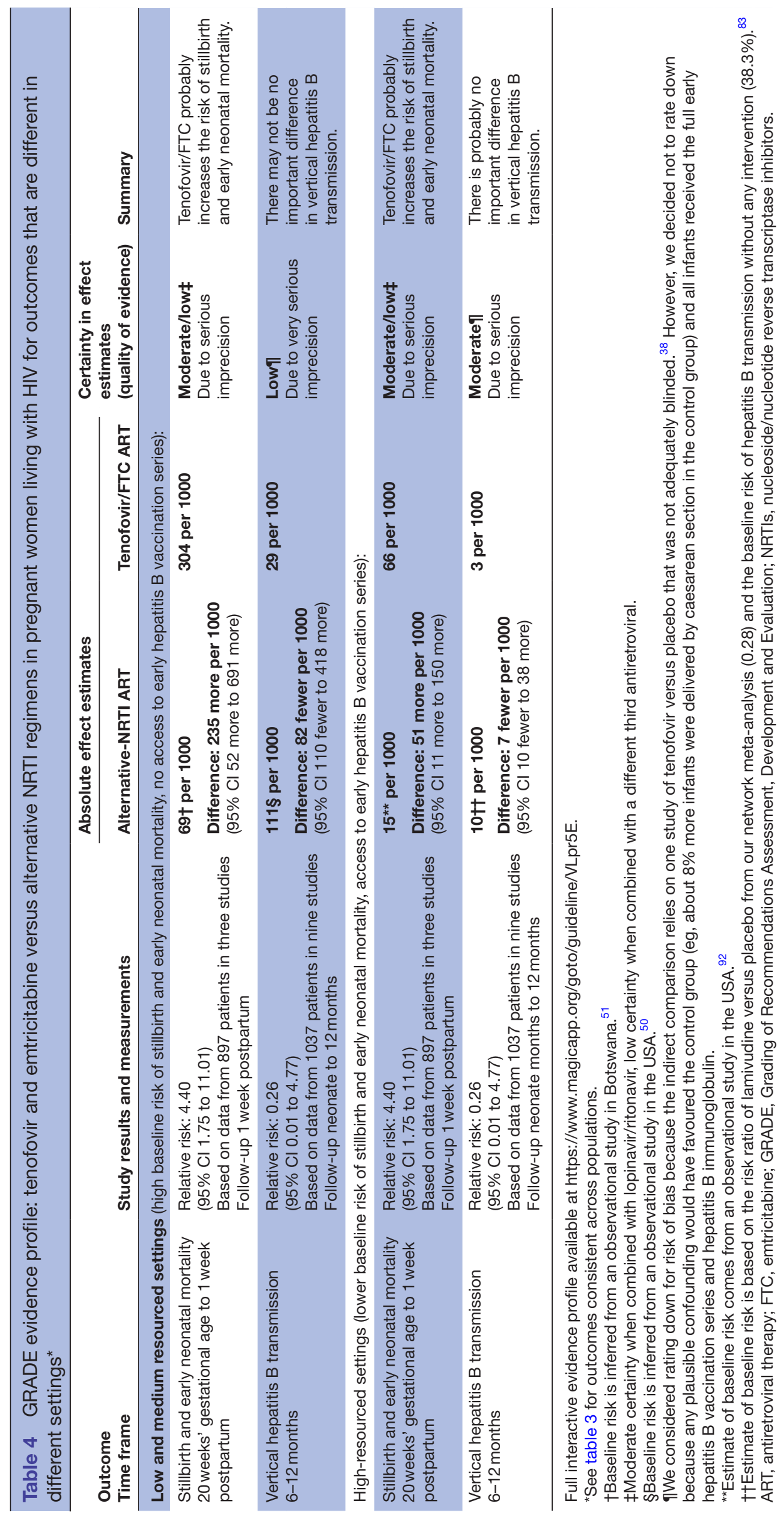


laboratory adverse events: $5.9 \%$ of 324 with AZT/lamivudine and $6.2 \%$ of 315 with tenofovir/FTC: RD 5 more per 1000, CI 24 fewer to 58 more (table 3).

\section{Medium and long-term development}

None of the studies reported medium and long-term developmental outcomes.

\section{Hepatitis B outcomes}

We screened 1035 titles and abstracts and ultimately included 33 comparative studies of NRTIs (tenofovir, lamivudine and FTC) in pregnant women living with HBV (online supplementary appendix $3 \mathrm{c}$ and online supplementary appendix 7). ${ }^{34} 3852-81$ The primary network meta-analysis was restricted to RCTs and included eight RCTs ( $\mathrm{n}=857$ ) comparing lamivudine with a control

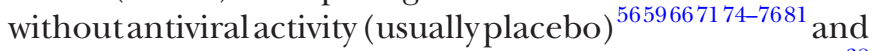
one RCT ( $n=180$ ) that compared tenofovir with placebo ${ }^{38}$ (online supplementary appendix 6). Two additional RCTs that compared lamivudine with placebo met inclusion criteria but were excluded from the analyses post hoc. ${ }^{34} 82$ One included 35 women with HIV and HBV coinfection, but no transmission events occurred in either group. ${ }^{34}$ The other, an unpublished study identified in the reference list of a systematic review, ${ }^{22}$ was excluded post hoc because the methods and definition of transmission were not described in sufficient detail. ${ }^{82}$ The main limitations within the RCTs were possible lack of allocation concealment (all but two RCTs) and lack of blinding (all but two RCTs) (online supplementary appendix 4b).

The secondary network meta-analysis included an additional 22 observational studies with an additional 1522 pregnant women, thus included 31 studies with 2559 pregnant women (online supplementary appendix 6). All 22 observational studies failed to adjust or match for most known confounders, the included populations were probably dissimilar in nine studies, and cointerventions may have been applied differently between the groups in 10 studies (online supplementary appendix 4c). Including the RCTs, 19 studies were conducted primarily in China, 8 in Europe or North America, and 1 in Africa.

\section{Vertical transmission of HBV}

There was low global heterogeneity for the network restricted to RCTs and for the network that included observational studies (global $\mathrm{I}^{2}=0 \%$ for both). There were no concerns of intransitivity. In the network restricted to RCTs (online supplementary appendix 8), lamivudine reduced risk of vertical transmission of HBV more than control (RR 0.28, CI 0.17 to 0.49; high certainty) as did tenofovir, but the CI included no effect (RR 0.07, CI 0.00 to 1.29) (table 4, figure 5). There was no apparent difference between tenofovir and lamivudine: RR 0.26, CI 0.01 to 4.77. Without antiviral therapy, the baseline risk of transmission is approximately 1 in $100^{83}$ in high-income countries and is approximately 380 per 1000 in low-resource countries without access to early neonatal hepatitis $\mathrm{B}$ vaccination and hepatitis B immunoglobulin (HBIg) ${ }^{84}$
The effect of tenofovir compared with lamivudine on vertical transmission of $\mathrm{HBV}$ in high-income countries is 7 fewer per 1000, CI 10 fewer to 38 more, and in low-income countries is 82 fewer per 1000, CI 110 fewer to 418 more (table 4).

When observational studies were included in the network meta-analysis (online supplementary appendix 9), tenofovir reduced risk of vertical transmission of hepatitis B compared with control: RR 0.23, CI 0.10 to 0.54; low certainty because of observational data (figure 5). Tenofovir did not reduce risk of vertical transmission of HBV compared with lamivudine: RR 0.99, 0.38 to 2.59; very low certainty due to observational data and imprecision (online supplementary appendix 9).

The pooled estimates of RCTs are shown in red, the pooled estimate of observational data is shown in yellow, and the pooled mixed estimates shown in blue.'

\section{Other hepatitis B outcomes}

Five studies (one RCT, four observational) reported hepatitis B flares, including three that compared tenofovir with control ${ }^{385763}$ and two that compared lamivudine with control. ${ }^{6872}$ Four studies defined a hepatitis flare as an increase in serum transaminase levels more than five times the upper limit of normal and one used an increase more than two times the upper limit of normal. ${ }^{72}$ All included flares prior to and after stopping antiviral therapy. The network had high global heterogeneity $\left(\mathrm{I}^{2}=63.5 \%, \mathrm{p}=0.042\right.$ for inconsistency; online supplementary appendix 10). We found no apparent difference between any of tenofovir, lamivudine or control, but our certainty in the evidence was very low for all comparisons due to observational data, inconsistency and very wide CIs (online supplementary appendix 10). One study reported the development of $\mathrm{HBV}$ resistance: $\mathrm{HBV}$ lamivudine resistance occurred in 1 of $25(4.0 \%, 0.1 \%$ to $20.4 \%$ ) women. ${ }^{53}$

\section{DISCUSSION}

The PROMISE trial dominated results for neonatal outcomes. ${ }^{12}$ We found moderate certainty evidence of a large absolute increase (point estimate 5\%) in stillbirth and early neonatal death with tenofovir/FTC versus AZT/lamivudine when they are combined with $\mathrm{LPV} / \mathrm{r}$. Moderate certainty evidence also suggested an increase in prematurity before 34 weeks with tenofovir/ FTC plus LPV/r versus AZT/lamivudine (point estimate also approximately $5 \%$ ). The evidence is indirect when applied to cART regimens in which the third antiretroviral is something other than $\mathrm{LPV} / \mathrm{r}$, particularly if it is not a protease inhibitor. In this situation, our certainty is low rather than moderate.

We also summarised the results of observational studies comparing tenofovir-based ART regimens with alternatives on stillbirth and neonatal deaths. Based on similar evidence, our review comes to a different conclusion than another recent meta-analysis. ${ }^{13}$ The reason for this 


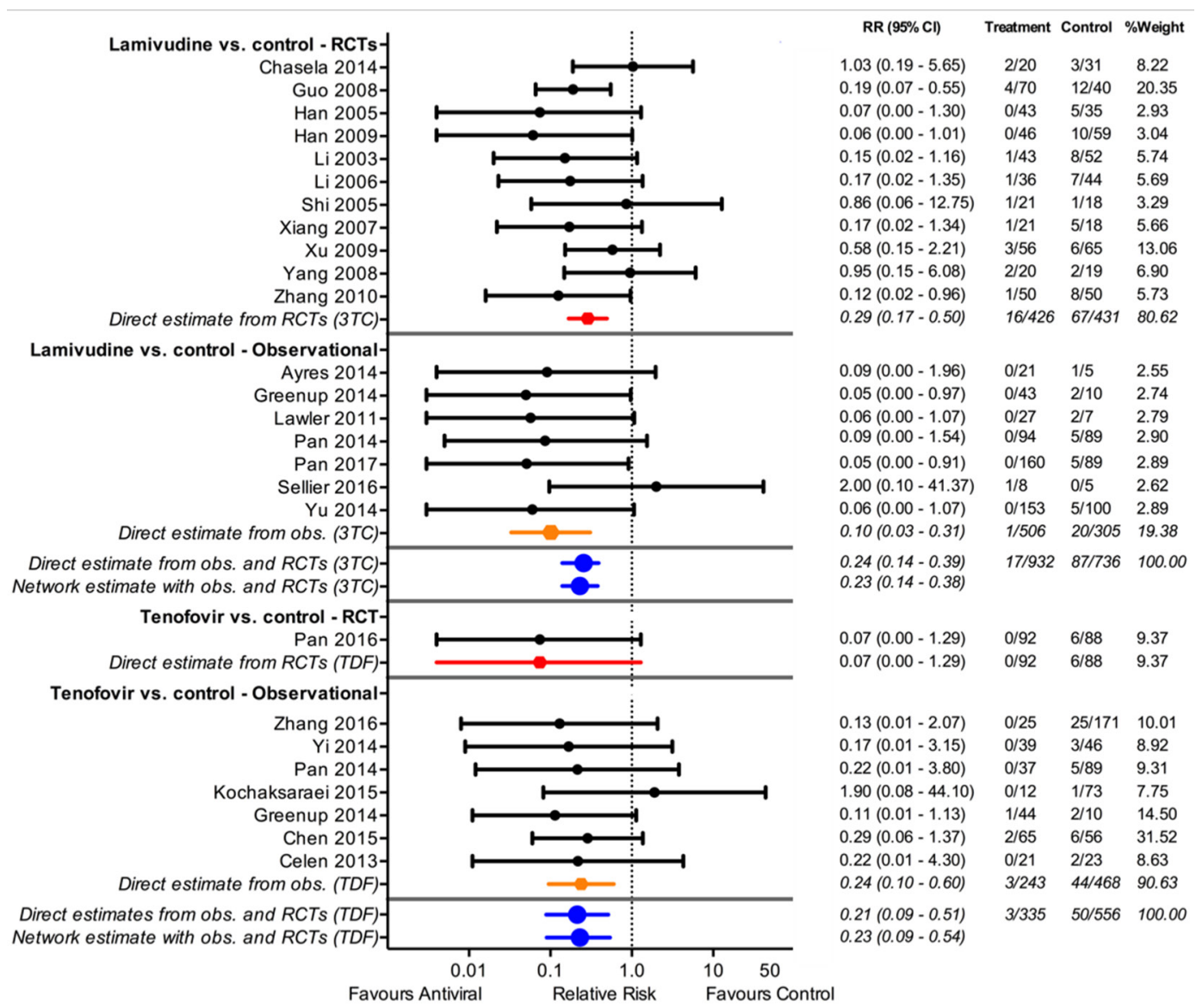

Figure 5 Forest plot of maternal antivirals (lamivudine and tenofovir) versus control (no antiviral) for prevention of vertical transmission of hepatitis B, by study type and antiviral. 3TC, lamivudine; ART, antiretroviral therapy; NRTI, nucleoside/nucleotide reverse transcriptase inhibitor; RCT, randomised controlled trial; RR, risk ratio; TDF, tenofovir disoproxil fumarate.

is Nachega and colleagues ${ }^{13}$ pooled RCTs and observational studies which, given the much higher certainty associated RCTs, we consider inadvisable and, indeed, inappropriate. This is particularly the case here because the available observational studies, already beginning as low quality using the GRADE framework ${ }^{85}$ were further limited by inconsistent results, imprecise pooled estimates and failure to adjust for important confounders. For instance, AZT/lamivudine is an older drug combination than tenofovir/FTC. Thus, clinical care for women who received AZT/lamivudine was more likely limited or outdated for other aspects of their pregnancy.

For the other key outcomes, there did not appear to be important differences between tenofovir-based regimens and alternatives. Outcomes without evidence of a between group difference include acceptability to pregnant women, clinical and laboratory maternal adverse events, maternal mortality, maternal viral load, vertical transmission of HIV, birth defects, low birth weight and prematurity prior to 37 weeks. Further, in pregnant women with hepatitis B coinfection, tenofovir and lamivudine likely confer a similar large reduction in risk of vertical hepatitis B transmission compared with no maternal intervention, although this is more certain for lamivudine than it is for tenofovir (high versus moderate certainty).
Strengths of our review include the comprehensive search; duplicate assessment of eligibility, risk of bias and data abstraction; summarisation of both RCT and observational studies; careful attention to what findings can or cannot be appropriately pooled; and use of the GRADE framework to address certainty of evidence. The primary limitations of the review are associated with the available evidence. The key results come primarily from a single study of moderate size. ${ }^{12}$ Single studies demonstrating large benefits on the basis of small number of events typically yield large overestimates of effect. ${ }^{86}$ This is likely true of harm outcomes as well, suggesting that the increase in stillbirths and neonatal deaths with tenofovir/FTC likely represents an overestimate. Because some have raised concerns that the event rates in the AZT/lamivudine arm are lower than might have been anticipated, we also performed a sensitivity analysis that includes participants in the AZT/lamivudine arm who were randomised early in the PROMISE study, before the tenofovir/FTC arm was added. $^{12}$

The results raise challenges in interpretation. The first is the mechanisms that tenofovir/FTC might increase in stillbirths and neonatal mortality. One hypothesis would be that the mediating factor is prematurity. Support from this hypothesis comes from the increase in prematurity 
before 34 weeks in the tenofovir group, and that approximately two-thirds of the deaths were attributed to prematurity or sequelae of prematurity.

Another interpretation issue is whether the culprit drug that might cause an increase in stillbirths/early deaths is tenofovir or FTC, and circumstances in which the culprit drug would lead to increases in stillbirths and neonatal deaths. The culprit could be tenofovir or FTC, or the combination of the two.

Another mechanism postulates a role for LPV/ $\mathrm{r}$ in the increase in stillbirths and neonatal deaths. ${ }^{87}$ This cannot be a direct effect: patients in both the tenofovir/FTC and AZT/lamivudine groups received LPV $/ \mathrm{r} .{ }^{12}$ Thus, the only possibility for implicating $\mathrm{LPV} / \mathrm{r}$ is that it modifies the effect of tenofovir/FTC but not AZT/lamivudine on stillbirths and neonatal mortality. Were this true, tenofovir/ FTC would have an adverse effect relative to AZT/lamivudine only when coadministered with $\mathrm{LPV} / \mathrm{r}$ or similar antiretrovirals. The mechanism of such an interaction is unlikely to be increased LPV drug levels in the presence of tenofovir: if anything, tenofovir decreases LPV drug levels. ${ }^{88-91}$ Further, protease inhibitors including LPV/r only slightly increase serum tenofovir levels ${ }^{8-91}$ and implicating this drug-drug interaction would nonetheless implicate tenofovir at serum concentrations within the typical therapeutic range (the increase in tenofovir from concurrent $\mathrm{LPV} / \mathrm{r}$ is a magnitude lower than normal variability between patients). ${ }^{92}$ The increased LPV/r dosing used in the PROMISE study during the third trimester provides similar serum drug concentrations to non-pregnant women taking $\mathrm{LPV} / \mathrm{r}^{93}$ Thus, the hypothesis that the adverse effects on fetal outcomes with tenofovir/FTC occur only with concomitant administration of $\mathrm{LPV} / \mathrm{r}$ has no evident biological basis. Nevertheless, we conservatively chose to rate down our certainty in the evidence for indirectness from moderate to low for key outcomes when tenofovir/FTC is combined with antiretrovirals other than $\mathrm{LPV} / \mathrm{r}$.

Tenofovir is currently the drug of choice for prevention of vertical transmission of HBV. The PROMISE results raise challenges for maternal prophylaxis against vertical transmission of HBV. The evidence that lamivudine results in a large reduction in vertical transmission of HBV compared with no antiviral therapy warrants higher certainty than is the case for tenofovir (although point estimates are similar). ${ }^{38}$ The results of our indirect comparisons suggest similar effects with use of tenofovir and lamivudine in decreasing vertical transmission of HBV. Generally, tenofovir is favoured for its lower likelihood of pretreatment HBV resistance and of developing HBV resistance during treatment than lamivudine. ${ }^{14}$ These considerations are particularly important in pregnant women who have long-term exposure to lamivudine, are at high risk of vertical transmission and in those who do not have access to early infant $\mathrm{HBV}$ vaccination or HBIg.

The results of this review present a dilemma for policymakers. Tenofovir/FTC is convenient as a single pill taken once a day. It is also the currently recommended regimen and allows harmonisation across a wide range of populations, resulting in simplification of cART and widespread provision in low-income and middle-income countries. Moreover, the adverse effect on stillbirths and neonatal mortality is likely an overestimate, and the mechanism and circumstances under which the effect exists remain uncertain. Nevertheless, fully informed pregnant women living with HIV are likely to choose regimens that do not include tenofovir or FTC.

\section{Author affiliations}

${ }^{1}$ Department of Health Research Methods, Evidence, and Impact, McMaster University, Hamilton, Canada

${ }^{2}$ Department of Medicine, University of Toronto, Toronto, Canada ${ }^{3}$ Department of Medicine, McMaster University, Hamilton, Canada ${ }^{4}$ Southern Alberta HIV Clinic, Alberta Health Services, Calgary, Canada

${ }^{5}$ The Infectious Diseases Society of America, Washington, USA

${ }^{6}$ Faculty of Medicine, University of Toronto, Toronto, Canada

${ }^{7}$ Gastroenterology and Hepatology Unit, College of Medicine, University of Lagos and Lagos University Teaching Hospital, Lagos, Nigeria

${ }^{8}$ Division of General Pediatrics, University Hospitals of Geneva, Geneva, Switzerland

${ }^{9}$ Faculty of Health Sciences, McMaster University, Hamilton, Canada

Acknowledgements We thank the members of the Rapid Recommendations panel for critical feedback on outcome and subgroup selection, GRADE judgements and manuscript feedback, including Susan Bewley (chair and obstetrician), Lyubov Lytvyn (BMJ Rapid Recommendation patient-liaison), Graham Taylor (professor of retrovirology), Rhonda Marama Mullen (patient representative), Florence Anam (patient representative), Teresia Otieno (patient representative), Claudia BeltránArroyave (paediatric infectious diseases specialist), Patrick Mbah Okwen (general practitioner), Nelly Mugo (obstetrician), Ruth Nduati (paediatrician), Henry Luma (infectious diseases specialist), Haresh Kirpalani (neonatologist), Thomas Agoritsas (internist and methodologist) and Per Vandvik (internist and methodologist).

Contributors RAS, GHG conceived the study idea. RAS and GHG wrote the first draft of the manuscript. PEA, RM, RAS designed the search strategy. RAS, FF, RM, PEA, AA, AM, YC, YZ, HM, EH, YL, DZ screened abstracts and full texts. RAS, FF, RM, $P E A, A A, A M, Y C, Y Z, H M, E H, Y L, D Z$ acquired the data and judged risk of bias in the studies. RAS and FF performed the data analysis. All authors interpreted the data analysis and critically revised the manuscript. RAS had full access to all of the data in the study, and takes responsibility for the integrity of the data and the accuracy of the data analysis. RAS is the guarantor.

Competing interests RAS, AM, YZ, and GHG are members of the GRADE Working Group. There are no other relationships or activities that could appear to have influenced the submitted work.

Provenance and peer review Not commissioned; externally peer reviewed. Data sharing statement All data are freely available within the appendices.

Open Access This is an Open Access article distributed in accordance with the Creative Commons Attribution Non Commercial (CC BY-NC 4.0) license, which permits others to distribute, remix, adapt, build upon this work non-commercially, and license their derivative works on different terms, provided the original work is properly cited and the use is non-commercial. See: http://creativecommons.org/ licenses/by-nc/4.0/

(c) Article author(s) (or their employer(s) unless otherwise stated in the text of the article) 2017. All rights reserved. No commercial use is permitted unless otherwise expressly granted.

\section{REFERENCES}

1. World Health Organization. Number of women living with HIV. Geneva, Switzerland: World Health Organization, 2017. http://www. who.int/gho/hiv/epidemic_status/cases_adults_women_children/en/ (accessed 20 Mar 2017).

2. Rates of mother-to-child transmission of HIV-1 in Africa, America, and Europe: results from 13 perinatal studies. The Working Group on 
Mother-To-Child Transmission of HIV. J Acquir Immune Defic Syndr Hum Retrovirol 1995;8:506-10.

3. De Cock KM, Fowler MG, Mercier E, et al. Prevention of motherto-child HIV transmission in resource-poor countries: translating research into policy and practice. JAMA 2000;283:1175-82.

4. UNAIDS. HIV Fact Sheets, 2017. http://aidsinfo.unaids.org/ (accessed 20 Mar 2017).

5. World Health Organization. Prevention of mother-to-child transmission: World Health Organization, 2017. Available from. http:// www.who.int/gho/hiv/epidemic_response/PMTCT_text/en/ (accessed 04 Nov 2017).

6. Forbes JC, Alimenti AM, Singer J, et al. A national review of vertical HIV transmission. AIDS 2012;26:757-63.

7. World Health Organization. WHO validates elimination of mother-tochild transmission of HIV and syphilis in Cuba, 2015.

8. Danel C, Moh R, Gabillard D, et al. A Trial of Early Antiretrovirals and Isoniazid Preventive Therapy in Africa. N Engl J Med 2015;373:808-22.

9. Lundgren JD, Babiker AG, Gordin F, et al. Initiation of Antiretroviral Therapy in Early Asymptomatic HIV Infection. N Engl J Med 2015;373:795-807.

10. World Health Organization. Consolidated guidelines on the use of antiretroviral drugs for treating and preventing HIV infection. 2nd edition, 2016

11. Siemieniuk RA, Mah Ming J, Guyatt GH, et al. Antiretroviral therapy for pregnant women living with HIV. BMJ. (Clinical research ed) (Cosubmitted).

12. Fowler MG, Qin M, Fiscus SA, et al. Benefits and Risks of Antiretroviral Therapy for Perinatal HIV Prevention. N Engl J Med 2016;375:1726-37.

13. Nachega JB, Uthman OA, Mofenson LM, et al. Safety of Tenofovir Disoproxil Fumarate-Based Antiretroviral Therapy Regimens in Pregnancy for HIV-Infected Women and Their Infants: A Systematic Review and Meta-Analysis. J Acquir Immune Defic Syndr 2017;76:1-12.

14. Terrault NA, Bzowej NH, Chang KM, et al. AASLD guidelines for treatment of chronic hepatitis B. Hepatology 2016;63:261-83.

15. Mugo NR, Hong T, Celum C, et al. Pregnancy incidence and outcomes among women receiving preexposure prophylaxis for HIV prevention: a randomized clinical trial. JAMA 2014;312:362-71.

16. UNAIDS. On the fast-track to end AIDS: UNAIDS 2016-2021 Strategy. 2015.

17. Fowler MG, Qin M, Fiscus SA, et al, 2015. PROMISE: Efficacy and Safety of 2 Strategies to Prevent Perinatal HIV Transmission. Conference on retroviruses and opportunistic infections. Seattle, USA (Abstract 31LB).

18. Panel on Treatment of HIV-Infected Pregnant Women and Prevention of Perinatal Transmission Recommendations for Use of Antiretroviral Drugs in Pregnant HIV-1-Infected Women for Maternal Health and Interventions to Reduce Perinatal HIV Transmission in the United States. 2016.

19. Lytvyn L, Siemieniuk RA, Guyatt GH, et al. Values and preferences of pregnant women living with HIV considering antiretroviral therapy. BMJ. (Co-submitted).

20. Siemieniuk RA, Agoritsas T, Macdonald $\mathrm{H}$, et al. Introduction to BMJ Rapid Recommendations. BMJ 2016;354:i5191.

21. Kanters S, Vitoria M, Doherty M, et al. Comparative efficacy and safety of first-line antiretroviral therapy for the treatment of HIV infection: a systematic review and network meta-analysis. Lancet HIV 2016;3:e510-20.

22. Brown RS, McMahon BJ, Lok AS, et al. Antiviral therapy in chronic hepatitis B viral infection during pregnancy: A systematic review and meta-analysis. Hepatology 2016;63:319-33.

23. Poolman RW, Agoritsas T, Siemieniuk RA, et al. Low intensity pulsed ultrasound (LIPUS) for bone healing: a clinical practice guideline. BMJ 2017;356:j576.

24. Vandvik PO, Otto CM, Siemieniuk RA, et al. Transcatheter or surgical aortic valve replacement for patients with severe, symptomatic, aortic stenosis at low to intermediate surgical risk: a clinical practice guideline. BMJ 2016;354:i5085.

25. Siemieniuk RAC, Harris IA, Agoritsas T, et al. Arthroscopic surgery for degenerative knee arthritis and meniscal tears: a clinical practice guideline. BMJ. In Press. 2017;357:j1982.

26. U.S. Department of Health and Human Services, National Institutes of Health, Division of AIDS National Institute of Allergy and Infectious Diseases Division of AIDS (DAIDS) Table for Grading the Severity of Adult and Pediatric Adverse Events. 2014. version 2.0 ed.

27. Busse JWG G. Modification of Cochrane tool to assess risk of bias in randomized trials 2013. https://distillercer.com/wp-content/uploads/ 2014/02/Tool-to-Assess-Risk-of-Bias-in-Randomized-ControlledTrials.docx (accessed July 11, 2016).
28. AkI EA, Sun X, Busse JW, et al. Specific instructions for estimating unclearly reported blinding status in randomized trials were reliable and valid. J Clin Epidemiol 2012;65:262-7.

29. Busse JWG G. Modification of Ottawa-Newcastle tool to assess risk of bias in observational studies 2013. (accessed 11 Jul 2016).

30. Guyatt GH, Oxman AD, Vist GE, et al. GRADE: an emerging consensus on rating quality of evidence and strength of recommendations. BMJ 2008;336:924-6.

31. Puhan MA, Schünemann $\mathrm{HJ}$, Murad MH, et al. A GRADE Working Group approach for rating the quality of treatment effect estimates from network meta-analysis. BMJ 2014;349:95630.

32. Jackson D, White IR, Riley RD. Quantifying the impact of betweenstudy heterogeneity in multivariate meta-analyses. Stat Med 2012;31:3805-20.

33. Spencer FA, lorio A, You J, et al. Uncertainties in baseline risk estimates and confidence in treatment effects. BMJ 2012;345:e7401.

34. Wang L, Wiener J, Bulterys M, et al. Hepatitis B Virus (HBV) Load Response to 2 Antiviral Regimens, Tenofovir/Lamivudine and Lamivudine, in HIV/ HBV-Coinfected Pregnant Women in Guangxi, China: The Tenofovir in Pregnancy (TiP) Study. $J$ Infect Dis 2016;214:1695-9.

35. Campbell TB, Smeaton LM, Kumarasamy N, et al. PEARLS study team of the ACTG. Efficacy and safety of three antiretroviral regimens for initial treatment of HIV-1: a randomized clinical trial in diverse multinational settings. PLoS Med 2012;9:e1001290.

36. Bunge K, Balkus J, Noguchi L, et al, 2015. Pregnancy incidence and outcomes in women receiving tenofovir-based PrEP in the VOICE trial. International AIDS conference. Vancouver, Canada (MOPEC480)

37. Van Damme L, Corneli A, Ahmed K, et al. Preexposure prophylaxis for HIV infection among African women. N Engl J Med 2012;367:411-22.

38. Pan CQ, Duan Z, Dai E, et al. Tenofovir to Prevent Hepatitis B Transmission in Mothers with High Viral Load. N Engl J Med 2016;374:2324-34

39. Gibb DM, Kizito H, Russell EC, et al. Pregnancy and infant outcomes among HIV-infected women taking long-term ART with and without tenofovir in the DART trial. PLoS Med 2012;9:e1001217.

40. Rough K, Seage GR, Williams PL, et al, 2017. TDF/FTC in pregnancy shows no increase in adverse infant birth outcomes in US cohorts. Conference on Retroviruses and Opportunistic Infections. Seattle, Washington (Abstract \#779).

41. Zash R, Jacobson D, Diseko M, et al, 2017. Adverse birth outcomes differ by ART regimen from conception in Botswana. Conference on Retroviruses and Opportunistic Infections. Seattle, Washington (Abstract \#25).

42. Antiretroviral Pregnancy Registry Steering Committee. 2016. Antiretroviral Pregnancy Registry International Interim Report for 1 January 1989 through 31 July 2016. Wilmington, USA.

43. Gallant JE, DeJesus E, Arribas JR, et al. Tenofovir DF, emtricitabine, and efavirenz vs. zidovudine, lamivudine, and efavirenz for HIV. N Engl J Med 2006;354:251-60.

44. Nishijima T, Takano M, Ishisaka M, et al. Abacavir/lamivudine versus tenofovir/emtricitabine with atazanavir/ritonavir for treatment-naive Japanese patients with HIV-1 infection: a randomized multicenter trial. Intern Med 2013;52:735-44.

45. Post FA, Moyle GJ, Stellbrink HJ, et al. Randomized comparison of renal effects, efficacy, and safety with once-daily abacavir/lamivudine versus tenofovir/emtricitabine, administered with efavirenz, in antiretroviral-naive, HIV-1-infected adults: 48-week results from the ASSERT study. J Acquir Immune Defic Syndr 2010;55:49-57.

46. Rey $\mathrm{D}$, Hoen $\mathrm{B}$, Chavanet $\mathrm{P}$, et al. High rate of early virological failure with the once-daily tenofovir/lamivudine/nevirapine combination in naive HIV-1-infected patients. J Antimicrob Chemother 2009;63:380-8.

47. Sax PE, Tierney C, Collier AC, et al. Abacavir/lamivudine versus tenofovir DF/emtricitabine as part of combination regimens for initial treatment of HIV: final results. J Infect Dis 2011;204:1191-201.

48. Sax PE, Tierney C, Collier AC, et al. Abacavir-lamivudine versus tenofovir-emtricitabine for initial HIV-1 therapy. N Engl J Med 2009;361:2230-40

49. Smith KY, Patel P, Fine D, et al. Randomized, double-blind, placebomatched, multicenter trial of abacavir/lamivudine or tenofovir/ emtricitabine with lopinavir/ritonavir for initial HIV treatment. AIDS 2009;23:1547-56.

50. Reddy UM, Laughon SK, Sun L, et al. Prepregnancy risk factors for antepartum stillbirth in the United States. Obstet Gynecol 2010;116:1119-26.

51. Chen JY, Ribaudo HJ, Souda S, et al. Highly active antiretroviral therapy and adverse birth outcomes among HIV-infected women in Botswana. J Infect Dis 2012;206:1695-705. 
52. Ayres A, Yuen L, Jackson KM, et al. Short duration of lamivudine for the prevention of hepatitis B virus transmission in pregnancy: lack of potency and selection of resistance mutations. J Viral Hepat 2014:21:809-17.

53. Ayres A, Yuen L, Manoharan S, et al. 736 lamivudine in late pregnancy for prevention of hbv transmission: Effectiveness and detection of antiviral resistance. J Hepatol 2011;54:S295-6.

54. Carey I, Lai JC CWW, et al. Preventing mother to child transmission of HBV in an ethnically diverse South London population: an effective multidisciplinary clinical pathway (Abstract). Hepatology 2016;63:S01.

55. Celen MK, Mert D, Ay M, et al. Efficacy and safety of tenofovir disoproxil fumarate in pregnancy for the prevention of vertical transmission of HBV infection. World J Gastroenterol 2013;19:9377-82.

56. Chasela CS, Kourtis AP, Wall P, et al. Hepatitis B virus infection among HIV-infected pregnant women in Malawi and transmission to infants. J Hepatol 2014;60:508-14.

57. Chen HL, Lee CN, Chang CH, et al. Efficacy of maternal tenofovir disoproxil fumarate in interrupting mother-to-infant transmission of hepatitis B virus. Hepatology 2015;62:375-86.

58. Greenup AJ, Tan PK, Nguyen V, et al. Efficacy and safety of tenofovir disoproxil fumarate in pregnancy to prevent perinatal transmission of hepatitis B virus. J Hepatol 2014;61:502-7.

59. Guo Y, Sx L, SI G, et al. Effect of lamivudine treatment combined with active-passive immunization on interrupting mother to infant transmission of HBV. Clinical Focus 2008;23:1730-1.

60. Han GR, Fang ZX, Zhao W, et al. Efficacy and safety of lamivudine treatment on preventing hepatitis $B$ virus vertical transmission in pregnant women. Chin J Infect Dis 2009;27:673-6.

61. Han ZH, Chen YH, Lw L, et al. Effect and safety of preventing HBV vertical transmission by lamivudine treatment. Chinese $\mathrm{J}$ Intern Med 2005; $44: 378$

62. Huang $\mathrm{H}, \mathrm{Wu} \mathrm{Q}$, Wang Y. Tenofovir Disoproxil: Effective and Safe in Prevention of Mother-to-child Transmission of Hepatitis B Virus. Hepatology 2014;60:1088A.

63. Samadi Kochaksaraei G, Castillo E, Osman M, et al. Clinical course of 161 untreated and tenofovir-treated chronic hepatitis B pregnant patients in a low hepatitis B virus endemic region. J Viral Hepat 2016;23:15-22.

64. Lawler J, Glass A, Chatterjee U, et al. Nucleot(s)ide analogues to prevent perinatal transmission of HBV: Lamivudine is effective but tenofovir may be better. J Gastro Hepato 2011;26:100.

65. Wf $L$, Jiang R, Wei $Z$, et al. Clinical effect and safety of lamivudine in interruption of chronic HBV maternal to infant transmission. Chin Hepatol 2006;11:106-7.

66. Li XM, Yang YB, Hou HY, et al. Interruption of HBV intrauterine transmission: a clinical study. World J Gastroenterol 2003;9:1501-3.

67. Pan C, Ta A, Zeng Z. Safety of Tenofovir Disoproxil Fumarate Treatment in Late Pregnancy in Highly Viremic Mothers with Chronic Hepatitis B. J American Gastro 2013;108:S156.

68. Pan CQ, Yi W, Liu M, et al. Lamivudine therapy during the second vs the third trimester for preventing transmission of chronic hepatitis $B$. $J$ Viral Hepat 2017;24:246-52.

69. Pan CQ, Lan B, Chan S, et al. Su1007 Tenofovir or Lamivudine Use in Late Pregnancy Safely Reduces Perinatal Transmission of Hepatitis B Virus in Real-Life Practice. Gastroenterology 2014;146:961.

70. Sellier P, Schnepf N, Amarsy R, et al. Hepatitis B virus (HBV) status of children born to HIV/HBV co-infected women in a French hospital: a cross-sectional study. J Int AIDS Soc 2014;17(4 Suppl 3):19632.

71. Shi MF, Xm L, He J, et al. Study of lamivudine in interruption of HBV intrauterine infection. Clin Med Chin 2005;21:77-8.

72. Tan PK, Chatterjee U, Glass A, et al. Lamivudine in pregnancy: impact on hepatitis flares and $\mathrm{HBeAg}$ seroconversion post partum. $J$ Gastro Hepato 2012;27:181.

73. Virine B, Osiowy C, Gao S, et al. Hepatitis B Virus (HBV) Variants in Untreated and Tenofovir Treated Chronic Hepatitis B (CHB)
Patients during Pregnancy and Post-Partum Follow-Up. PLoS One 2015;10:e0140070.

74. Xiang GJ, Sun JW, Jiang SQ, et al. Evaluation of therapeutic effect in HBV vertical transmission by lamivudine treatment combined with active-passive immunization for pregnant women. Chin Prac Med 2007;2:14-16.

75. Wm X, Cui YT, Wang L, et al. Lamivudine in late pregnancy to prevent perinatal transmission of hepatitis B virus infection: a multicentre, randomized, double-blind, placebo-controlled study. J viral hepatitis 2009;16:94-103.

76. Yang S, Liu M, Wang L. Effect of high viral hepatitis B virus DNA loads on vertical transmission of hepatitis $B$ virus in late-pregnant women]. Zhonghua fu chan ke za zhi 2008;43:329-31.

77. Yi W, Pan CQ, Liu M, et al. Clinical outcomes of tenofovir disoproxil fumarate (TDF) treatment versus no treatment for pregnant women with active chronic hepatitis $\mathrm{B}(\mathrm{CHB})$ and elevated alanine aminotransaminase (ALT). Hepatology 2014;60:1094A.

78. $\mathrm{Mm} \mathrm{Y,} \mathrm{Jiang} \mathrm{Q,} \mathrm{Ji} \mathrm{Y,} \mathrm{et} \mathrm{al.} \mathrm{Comparison} \mathrm{of} \mathrm{telbivudine} \mathrm{versus}$ lamivudine in interrupting perinatal transmission of hepatitis $B$ virus. $J$ Clin Virol 2014:61:55-60.

79. Zhang H, Pan CQ, Pang Q, et al. Telbivudine or lamivudine use in late pregnancy safely reduces perinatal transmission of hepatitis $B$ virus in real-life practice. Hepatology 2014;60:468-76.

80. Zhang Y, Wu Q, Huang H, et al. J Hepatol 2016;64:S607 (Abstract FRI-154).

81. Zhang YF. The clinical observation of effect of lamivudine on interrupting mother to infant transmission of chronic HBV on 50 mothers. J Prat Obst Gynecol 2010;26:367-8.

82. Shi Z, Li X, Yang Y, et al. Clinical research on the interruption of mother to child transmissionof HBV- a randomized, double-blind, placebo-control study. 2008.

83. Schillie $\mathrm{S}$, Walker T, Veselsky $\mathrm{S}$, et al. Outcomes of infants born to women infected with hepatitis B. Pediatrics 2015;135:e1141-7.

84. Keane E, Funk AL, Shimakawa Y. Systematic review with metaanalysis: the risk of mother-to-child transmission of hepatitis $B$ virus infection in sub-Saharan Africa. Aliment Pharmacol Ther 2016;44:1005-17.

85. Guyatt GH, Oxman AD, Kunz R, et al. What is "quality of evidence" and why is it important to clinicians? BMJ 2008;336:995-8.

86. Montori VM, Devereaux PJ, Adhikari NK, et al. Randomized trials stopped early for benefit: a systematic review. Jama 2005;294:2203-9.

87. World Health Organization. Press Release: First PROMISE study results confirm WHO recommendations to treat pregnant women and reduce mother-to-child-transmission of HIV. 2014 http://www.who. int/hiv/mediacentre/news/promise-study-result/en/ (accessed $28 \mathrm{Jul}$ 2017).

88. Lack of clinically relevant drug-drug interactions between tenofovir DF and efavirenz, indinavir, lamivudine and lopinavir/ritonavir in healthy subjects. 8th European Conference on Clinical Aspects and Treatment of HIV Infection.

89. Pharmacokinetic drug interaction of lopinavir/ritonavir in combination with tenofovir in experienced HIV+ patients [abstract A-445]. 44th Interscience Conference on Antimicrobial Agents and Chemotherapy.

90. Comparison of lopinavir/r plasma levels with and without tenofovir as part of HAART in HIV-1 infected patients. 5th International Workshop on Clinical Pharmacology of HIV Therapy.

91. Kearney BP, Mathias A, Mittan A, et al. Pharmacokinetics and safety of tenofovir disoproxil fumarate on coadministration with lopinavir/ ritonavir. JAIDS 2006;43:278-83.

92. Kearney BP, Mathias A, Mittan A, et al. Pharmacokinetics and safety of tenofovir disoproxil fumarate on coadministration with lopinavir/ ritonavir. J Acquir Immune Defic Syndr 2006;43:278-83.

93. Best BM, Stek AM, Mirochnick M, et al. Lopinavir tablet pharmacokinetics with an increased dose during pregnancy. J Acquir Immune Defic Syndr 2010;54:381-8. 